\title{
The Density of the Lunar Surface Soil
}

\author{
RoNaLd F. ScOTT \\ California Institute of Technology, Pasadena, California 91109
}

Until the landing of the Surveyor 1 spacecraft on the moon, it had been inferred from earth-based optical, thermal, radio, and radar observations [Kopal, 1962; Salisbury and Glaser, 1964] that the surface of the moon consisted of material of low-bulk density (less than $1 \mathrm{~g} / \mathrm{cm}^{\mathrm{s}}$ ) and high porosity. Recently, a change in this belief, at least with respect to the optical data, has occurred [KenKnight et al., 1967], although a low density continues to be postulated on the basis of radiometric data [Campbell et al., 1968].

From the landing of Surveyor 1 in June 1966, I deduced that the mechanical resistanee of the lunar soil to the penetration of the Surveyor footpads was best explained [Christensen et $a l ., 1967]$ by the presence of a material of essentially 'normal' terrestrial soil properties, including density (approximately $1.5 \mathrm{~g} / \mathrm{cm}^{3}$ ).

The most direct measurement from the Surveyor 1 landing of a lunar soil property is the strength of the material. The strength of a soil material derives from four fundamental parameters: cohesion, angle of internal friction, density, and gravity [Scott, 1963]. The part contributed by cohesion is independent of the gravitational field, whereas the friction angle, the product of density, and the acceleration due to gravity (unit weight) are interrelated in their effect on the strength. I placed certain bounds on the cohesion of the lunar surface from the Surveyor 1 landing information [Christensen et al., 1967]; therefore the rest of the strength had to be accounted for by the friction-angle and unit-weight combination. Angles of internal friction of $30^{\circ}-40^{\circ}$ were in the right range to describe the Surveyor 1 landing and were compatible with the side slope conditions in the various small craters visible to Surveyor. For this range of angles, the density required to explain the strength was approximately $1.5 \mathrm{~g} / \mathrm{cm}^{2}$.

The Luna 13 spacecraft carried a gamma radiation instrument for surface density measurement to a depth of $15 \mathrm{~cm}$ and a penetration device for measuring the strength of the lunar surface. Following the landing of Luna 13 on the moon in December 1966, Tass [1966] reported that the gamma radiation measurement '. . . accords with a density not exceeding one gram per cubic centimeter' without elaborating the details. From the dynamics of the Luna 13 landing it was also reported [Tass, 1966] that comparison with tests on earth '. . . warrants the assumption that the mechanical properties of the moon's surface layer 20-30 centimeters deep are close to the properties of medium-density terrestrial soil.' The Russian investigators established, therefore, two values of density that were inconsistent.

In April 1967, Surveyor 3 landed on the moon carrying the surface sampler apparatus, for which I was principal investigator. Because of changes in the design of the device, necessitated by the spacecraft schedule, and because of the faulty telemetry received from the landed spacecraft, a direct measurement of the lunar surface density was not possible. However, the density can be calculated from the results of the experiments performed. In addition, the porosity (ratio of volume of voids between the particles to total volume) of the surface material can be estimated within fairly close limits from the nature of the deformations of the soil observed, as discussed below. Again, the density appears to be in the region of $1.5-2.0 \mathrm{~g} / \mathrm{cm}^{\mathrm{s}}$, and the porosity appears to be in the range of $0.35-0.45$ [Scott and Roberson, 1967]. The strength, porosity, density, cohesion, and angle of friction of the top few centimeters of the lunar soil are all values compatible with the behavior of an essentially ordinary, slightly cohesive earth soil of a wide range of grain sizes, but finer sizes (tens of microns in diameter and less) pre- 
dominate in a fairly compact state under lunar gravity. The lunar soil exhibited cohesion even after disturbance and also adhered to the bucket of the surface sampler; therefore the cohesion cannot be entirely attributed to cementing (of whatever nature) between the particles, although some cementing is undoubtedly present.

At this stage, it was difficult to reconcile my interpretation of the lunar surface density with the density value obtained directly by the Russian gamma radiation measurement. At the Cospar conference in London in July 1967, however, a paper was presented [Cherkasov et al., 1967] in which more details of the Russian experiments were given. It appears that the calibration of the gamma radiation device was not single-valued. In the words of the paper, 'The relationship between the intensity of recorded scattered radiation and the soil density with a given measuring scheme is shown by a parabolic curve turned by its protuberance upward [sic] and having a maximum with the bulk density of $1.5 \mathrm{~g} / \mathrm{cm}^{3}$. The ascending branch of the curve relates to the materials whose density is from 0.1 to 1.5 and the descending branch from 1.5 to $3.0 \mathrm{~g} / \mathrm{cm}^{3}$ (in earth's units). Therefore two values of the density correspond to any index of the counters-lower and greater than $1.5 \mathrm{~g} / \mathrm{cm}^{3}$. All data available are indicative of the fact that the presence of materials with the density over $1.5 \mathrm{~g} / \mathrm{cm}^{3}$ on the lunar surface is of little probability. Therefore the density along the ascending branch of the curve was taken into account.' Regarding the measurements made, the paper reports, "The intensity of scattered radiation recorded by the radiation densimeter sensor, taking into account gamma-radiation background on the moon corresponds to the bulk density of the surface layer $0.8 \mathrm{~g} / \mathrm{cm}^{3}$ on the ascending branch of the calibration curve or $2.1 \mathrm{~g} / \mathrm{cm}^{3}$ on the descending branch. Discarding the last figure as of little probability we come to the bulk density of $0.8 \mathrm{~g} / \mathrm{cm}^{3}$ indicating the existence of light, granular porous material.' No indication is given of the uncertainty in these figures. The remark in the first of these two quotations, 'all data available,' presumably refers to previous interpretations of optical, thermal, radio, and radar observations. It should be noted again that the density was obtained as an average to a depth of $15 \mathrm{~cm}$.

The Russian Luna 13 device for estimating the mechanical properties of the lunar surface material consisted of a cone penetrometer $3.5 \mathrm{~cm}$ in diameter pushed into the lunar surface a few centimeters by a small rocket giving $5-7$ $\mathrm{kg}$ of thrust for $0.6-1.0$ second. Only the penetration was measured, and the result was consistent with the penetration estimated for such a device in the lunar soil deduced from the Surveyor tests. The strength of the lunar material at the Luna 13 and Surveyor Ianding sites is therefore similar. With only this penetration information, it is not possible to deduce the nature of the soil resisting the penetration or to make much of an analysis. All that can be done [see also Cherkasov et al., 1967] is to push a similar device into a variety of model soils under similar conditions (vacuum, low gravitational acceleration) on earth. There is an infinite variety of soils that will give the same penetration for the same device under the same loading conditions; hence the terrestrial tests cannot indicate the cohesion or the friction angle of the lunar soil. However, because the gamma radiation instrument gave a reading that was interpreted as arising from a density of $0.8 \mathrm{~g} / \mathrm{cm}^{2}$, the investigators turned their attention to low density materials.

From the model soils of their earth tests, the Russian investigators came to the conclusion that the appropriate terrestrial analog to the lunar soil is a predominantly cohesive material, and, in fact, they describe it as being cemented. Cherkasov et al. [1967] remark, 'Summing up the data obtained from all three instruments, we may conclude that at the Luna 13 landing site the lunar surface seems to represent [sic] a layer of granular, loose, weakly cemented material, consisting of grains and granules of porous mineral, which are weakly interconnected at contact points.'

If a granular material is constructed of solid grains according to this specification, with a density of $0.8 \mathrm{~g} / \mathrm{cm}^{3}$ and a strength compatible with the lunar observation, it compresses in volume under loads applied to its surface. In particular, if a flat-based penetration device, like the surface sampler, is forced into such a soil, a hole is punched into the material, with little or no disturbance of the soil adjacent to 
the penetrometer. This behavior was observed neither with the surface sampler tests on Surveyor 3 [Scott and Roberson, 1967] nor accompanying the penetration of Surveyor footpads [Christensen et al., 1967]. Instead, when the 2.54-cm-wide surface sampler was forced into the lunar soil to a depth of $1.27 \mathrm{~cm}$, the adjacent surface bulged upward and cracked to a distance of $13-15 \mathrm{~cm}$ from the edge of the sampler. This indicated that little or no volume decrease of the lunar soil occurred during penetration by the sampler; therefore it was necessary for the sampler to displace the soil laterally. Terrestrial experiments indicate that, to exhibit this behavior, a soil must have a fairly low intergrain porosity, of the order of $0.35-0.45$. The porosity estimate from the Surveyor 3 data [Scott and Roberson, 1967] was made on this basis. Such a porosity is compatible with a bulk density of $1.5-2.0 \mathrm{~g} / \mathrm{cm}^{3}$ if the soil grains are solid and consist of minerals of common, terrestrially observed densities.

The conclusions from the Luna 13 and Surveyor investigations may be made more compatible if the grains of lunar soil are also porous as well as strong. It is not known if this is the case in the maria, but the possibility seems to be minimized in the Tycho ejecta blanket area because the surface sampler determination of the density of a rock fragment is in the range of $2.4-3.1 \mathrm{~g} / \mathrm{cm}^{3}$. The soil behavior near Tycho was only slightly different from that in the maria [Scott and Roberson, 1968].

Additionally, the low-density soil indicated by the Russian investigations would have to possess a cohesion considerably greater than that deduced from the Surveyor measurements to account for its strength.

If the higher value of lunar surface density (2.1 $\mathrm{g} / \mathrm{cm}^{8}$, with some leeway depending on the calibration of the device for different materials) is taken from the Luna 13 gamma radiation results, the soil behavior under their spacecraft's probe can be explained by the soil I postulate as existing at the Surveyor 3 site. I suggest that this is the case and that the lunar surface material to a depth of a few centimeters at the Surveyor 1, Luna 13, Sur- veyor $3,5,6$, and 7 landing sites consists of granular material whose density is in the range of densities of terrestrial soils, i.e. $1.5-2.0 \mathrm{~g} / \mathrm{cm}^{8}$. If a lower-density soil material is present, the surface sampler tests indicate that it can have a thickness of 1 or $2 \mathrm{~mm}$ at most. These tests also show an increase of strength with depth, possibly due to a density increase.

Acknowledgment. I am indebted to L. D. Jaffe of the Jet Propulsion Laboratory for giving me a copy of the Cherkasov et al. [1967] paper.

\section{REFERENCES}

Campbell, M. J., J. Ulrichs, and T. Gold, Density of the lunar surface, Science, 159, 3818, 1968.

Cherkasov, I. I., A. L. Kemurdzhian, L. N. Mikhailov, V. Mikheev, A. A. Morozov, A. A. Musatov, I. A. Savenko, M. I. Smorodinov, and V. V. Shvarev, Determination of the density and mechanical strength of the surface layer of the lunar crust at the landing site of Luna 13 automatic lunar landing station, paper presented at the 10th plenary meeting of Cospar, London, July 1967; published in Kosmicheskie Issledovaniia, 5, 746-757, 1967.

Christensen, E. M., S. A. Batterson, H. E. Benson, C. E. Chandler, R. H. Jones, R. F. Scott, E. N. Shipley, F. B. Sperling, and G. H. Sutton, Lunar surface mechanical properties-Surveyor $1, J$. Geophys. Res., 72, 801, 1967.

KenKnight, C. E., D. L. Rosenberg, and G. K. Wehner, Parameters of the optical properties of the lunar surface powder in relation to solarwind bombardment, J. Geophys. Res., 7 2,3105 , 1967.

Kopal, Z., Physics and Astronomy of the Moon, Academic Press, New York, 1962.

Salisbury, J. W., and P. E. Glaser, Eds., The Lunar Surface Layer, Academic Press, New York, 1964.

Scott, R. F., Principles of Soil Mechanics, chp. 8, Addison-Wesley, Reading, Massachusetts, 1963.

Scott, R. F., and F. I. Roberson, Soil mechanics surface sampler: Lunar surface tests and analyses, Surveyor 3 mission report, 2, scientific results, Calif. Inst. Technol. Jet Propulsion Lab. TR 32-1177, 1967.

Scott, R. F., and F. I. Roberson, Soil mechanics surface sampler: Surveyor 7 mission report, 2, scientific results, to be published, California Institute of Technology, Jet Propulsion Laboratory, 1968.

Tass International News Service, Moscow, December 20, 1966.

(Received April 11, 1968.) 